\title{
Target Recognition Based on CBR in Optoelectronic Hybrid System
}

\author{
Guangle $\mathrm{YaO}^{1,2, a}$, Qinzhang $\mathrm{Wu}^{1}$, Jiandan Zhong ${ }^{1,2, \mathrm{~b}}$, Kelin Sun ${ }^{1}$ \\ ${ }^{1}$ Institute of Optics and Electronics Chinese Academy of Sciences, Chengdu 610209, China \\ ${ }^{2}$ University of Electronic Science and Technology of China, Chengdu 610054, China \\ ayaoguangle@163.com, bzjdwell@163.com
}

Keywords: CBR, target recognition, KNN, expert system.

\begin{abstract}
Optoelectronic hybrid system is widely used in military field and civilian field. Target recognition is one of the important functions of optoelectronic hybrid system. In order to improve the system's flexibility and intelligence, expert system is involved into optoelectronic hybrid system. This paper presents a model of target recognition based on CBR (case-based reasoning) expert system in optoelectronic hybrid system. And this paper processes an experiment about target recognition to recognize the vehicles. In this experiment, the KNN (K-nearest neighbors), a classical algorithm of CBR and one UCI data-set is employed. Results demonstrate that CBR expert system can be used to recognize the target in optoelectronic hybrid system, and the accuracy and efficiency can be improved by selecting features.
\end{abstract}

\section{Introduction}

Optoelectronic hybrid system which is composed of Optoelectronic sensors, computer system and other equipment is used to detect, track, extract and recognize the target.

In order to improve the flexibility and intelligence, expert system is involved into the optoelectronic hybrid system. As one branch of expert system, CBR [1, 2] is the process of solving new problems based on the solutions of similar past cases. And CBR is not only a powerful method for computer reasoning, but also a pervasive behavior in everyday human problem solving; or, more radically, that all reasoning is based on past cases personally experienced. CBR is widely used for classification: it begins with a set of cases; each case is described in terms of a set of features; some classification algorithms are applied to the features' values of the cases. The weighted features selection, past cases and reasoning algorithm are playing important roles in efficiency and accuracy of reasoning.

This paper presents a model of target recognition based on CBR (case-based reasoning) expert system in optoelectronic hybrid system, and introduces an experiment using KNN algorithm. And weighted features selection is applied in experiment to improve the efficiency and accuracy of recognition.

\section{The Model of Target Recognition Based on Expert System}

Target recognition in Optoelectronic hybrid system is implemented by modules: image capturing, image pre-processing, target extraction, features extraction and expert system of target recognition. Fig 1 is the processing flow of target recognition by CBR expert system. And the module expert system of target recognition is the main point of this paper.
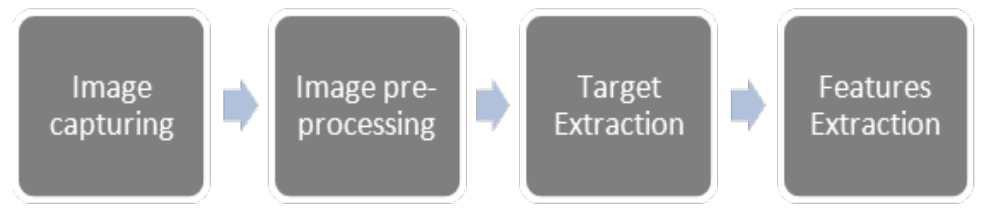

Fig. 1 The flow diagram of target recognition 
Image capturing module: This module converts an optical image into an electronic signal. It is used mostly in digital cameras, and other imaging devices. Electronic signal such as photo and video includes the target's images which reflect the feature of shape, silhouettes, texture and size et al.

Image pre-processing module: This module is used to improve the quality and reliability of images from image capturing module by some image processing algorithms, such as binning, normalization, filter and image plane separation.

Target extraction module: This module refers to the use of some image processing algorithms to map the locations of a target in the image.

Features extraction module: This module extracts the features of the target: shape, spectral signature, silhouettes, color or texture. In the experiment of this paper, the silhouettes features of target are prepared for expert system module.

Expert system module of target recognition: This module is the main point of this paper. In this module, the features of target are processed by expert system algorithm to recognize target. This module includes: interference engine and cases storage. Learning or reasoning algorithm in interference engine interacts with past cases in case storage to resolve the problem. The past cases with features' values are stored in case storage.

\section{CBR and KNN}

CBR [1, 2] is a branch of expert system and is the process of solving new problems based on the solutions of similar past cases. KNN [3], a well-known algorithm for CBR, is commonly based on the Euclidean Distance [4] between a test case and the past cases in case storage. Let $\mathrm{x}_{\mathrm{i}}$ be a test case with $\mathrm{p}$ features $\left(\mathrm{x}_{\mathrm{i} 1}, \mathrm{x}_{\mathrm{i} 2} \cdots \mathrm{x}_{\mathrm{ip}}\right)$, $\mathrm{p}$ the total number of features. The Euclidean Distance between test case $\mathrm{x}_{\mathrm{i}}$ and past case $\mathrm{x}_{\mathrm{j}}$ is defined as

$$
\operatorname{Dis}\left(x_{i}, x_{j}\right)=\sqrt{\sum_{k=1}^{p}\left(x_{i k}-x_{j k}\right)^{2}}
$$

The test case [5] is classified by a majority vote of its neighbors, with the test case being assigned to the class most common among its $\mathrm{K}$ nearest neighbors (the past cases with the top $\mathrm{K}$ smallest Dis (xi, $\mathrm{xj}$ ). If $\mathrm{K}=1$, then the target is simply assigned to the class of that single nearest neighbor. In Fig 2 , ' ' and '*' are two class past cases, ' 0 ' is the test case. The $\mathrm{K}=9$ nearest neighbors in the dotted circle are the candidate case, according the KNN, 'o' is assigned to ' $\bullet$ '.

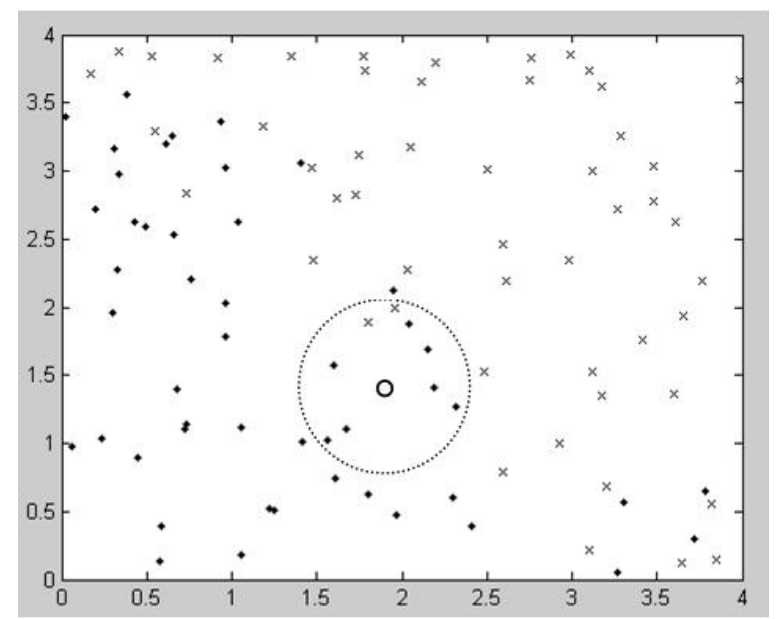

Fig. 2 K-nearest neighbor algorithm Schematic diagram

In KNN algorithm, every feature have different weights to the reasoning, a slice of features have small contribution, even negative effect to reasoning. Consequently the weighted features selecting can improve the efficiency and accuracy of reasoning. Lots of methods can be used to select features: information entropy, genetic algorithm. In the paper, a traversing experiment is used to select features. 
For a heavy-weighted feature, the accuracy of reasoning would be improved when delete it from reasoning process. So in the traversing experiment, each feature will be deleted in turn, to try to select the heavy weighted feature.

\section{Experiments}

To evaluate the model of target recognition based on expert system, a target recognition experiments was applied. And a vehicle silhouettes dataset in UCI machine learning repository [6] is employed in this experiment. The dataset comes from the Turing Institute, Glasgow, Scotland. Vehicles are viewed from different angles, and by image processing system 18 silhouettes features are extracted: COMPACTNESS, CIRCULARITY, DISTANCE CIRCULARITY, RADIUS RATIO, PR. AXIS ASPECT RATIO, MAX. LENGTH ASPECT RATIO and SCATTER RATIO et al.

In this experiment, we selected two kinds of vehicles (Chevrolet van and Saab 9000) cases to verify the target recognition based on CBR, divided these cases into two parts. One part includes 184 cases as past case in CBR, and the other part includes 232 cases as testing cases.

Before the recognition experiment, the traversing experiment described in previous chapter is processed to select weighted features. And in this recognition experiment we try to the recognize vehicle with all the features, the top 14 weighted features and the top 10 weighted features.

Fig 3 is the result of all vehicles recognition; Fig 4 is the result of only Saab recognition; Fig 5 is the result of the only Van recognition. These figures show that the accuracy of the recognition is from 75 percent to 100 percent using KNN with different K. And after weighted feature selection, the accuracy will be improved.

Fig 6 is the execution time of the recognition, and it shows that the execution time of the recognition will be reduced after weighted feature selection, and efficiency of recognition can be improved by weighted feature selection.

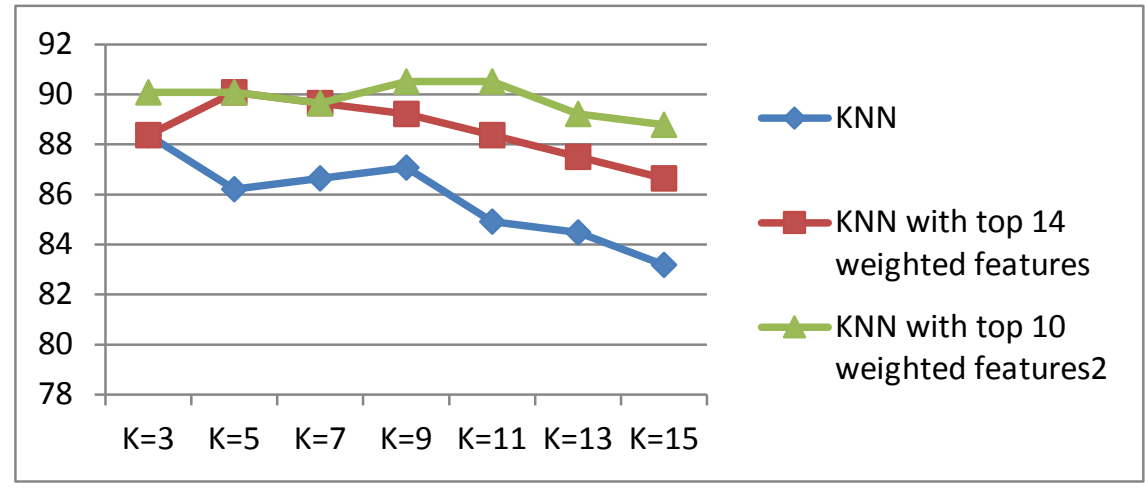

Fig. 3 Percentage of vehicles recognition accuracy

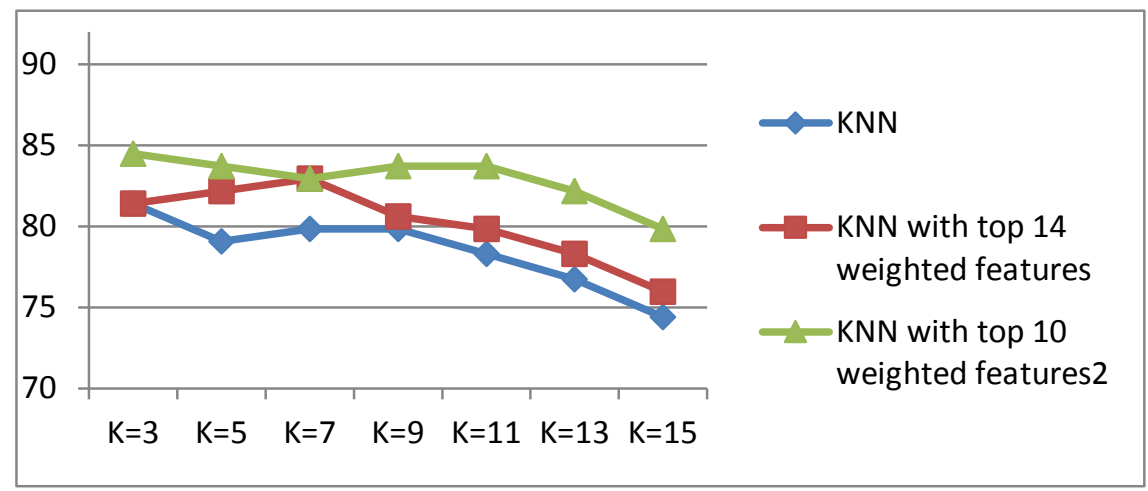

Fig. 4 Percentage of Saab recognition accuracy 


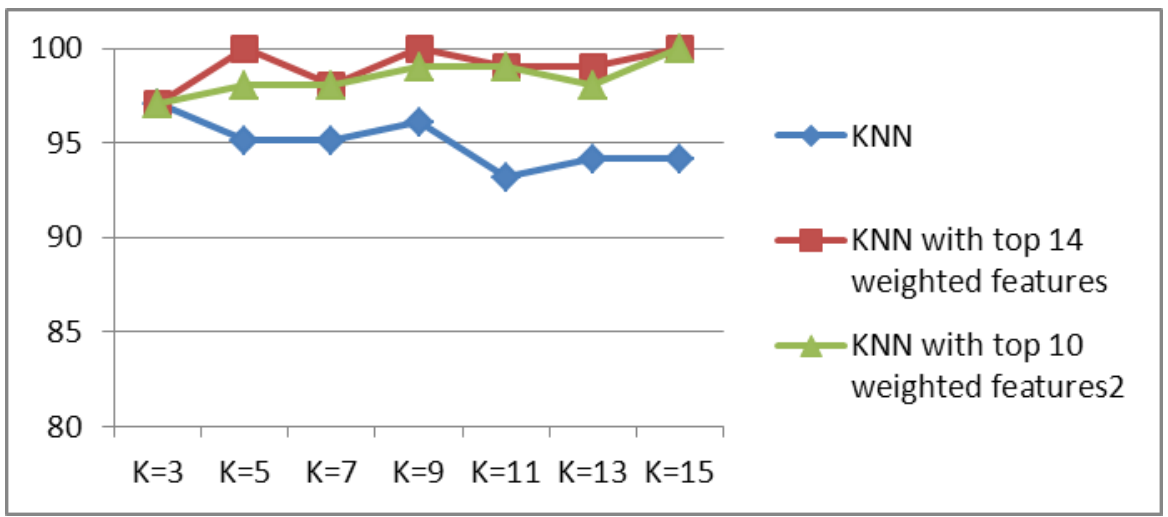

Fig. 5 Percentage of van recognition accuracy

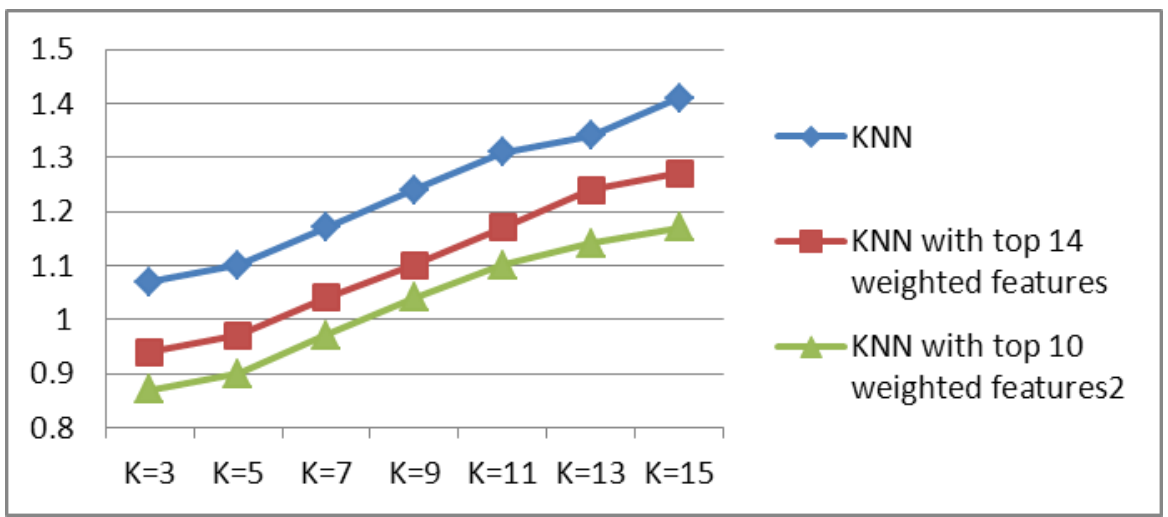

Fig. 6 Execution time (millisecond) of the recognition

\section{Summary}

This paper introduces a general target recognition model based on CBR in optoelectronic hybrid system. KNN of the CBR and weighted feature selection based on traversing experiments are introduced in this paper. Finally, a vehicle recognition experiment shows that vehicles can be recognized by CBR with its silhouettes features, and after weighted feature selection, the efficiency and accuracy can be both improved.

In the next step, more vehicles will be added to the vehicles dataset, and more CBR algorithm will be employed in this system model. The studying of the system will focus on the flexibility, intelligence, efficiency and accuracy.

\section{References}

[1] Kolodner J. L., Case-based reasoning, San Mateo, CA : Morgan Kaufman(1993)

[2] Leake B., CBR in Context: The Present and Future. In Leake D., Case-Based Reasoning: Experiences, Lessons, and Future Directions, AAAIPress/MITPress. (1996)

[3] Information on http://www.scholarpedia.org/article/K-nearest_neighbor

[4] Haixuan Yang, Machine Learning models on random graphs, Thesis for degree of doctor (2005)

[5] Chonghua Xu, Personal Recommendation Using a Novel Collaborative Filtering Algorithm in Customer Relationship Management, Discrete Dynamics in Nature and Society(2013)

[6] Information on http://www.ics.uci.edu/ mlearn/MLRepository.html 